\title{
Anxiety and attentional bias in preschool- aged children: an eyetracking study
}

Article

Accepted Version

Dodd, H., Hudson, J., Williams, T., Morris, T., Lazarus, R. and Byrow, Y. (2015) Anxiety and attentional bias in preschoolaged children: an eyetracking study. Journal of Abnormal Child Psychology, 43 (6). pp. 1055-1065. ISSN 0091-0627 doi: https://doi.org/10.1007/s10802-014-9962-x Available at https://centaur.reading.ac.uk/38941/

It is advisable to refer to the publisher's version if you intend to cite from the work. See Guidance on citing.

To link to this article DOI: http://dx.doi.org/10.1007/s10802-014-9962-x

Publisher: Springer

All outputs in CentAUR are protected by Intellectual Property Rights law, including copyright law. Copyright and IPR is retained by the creators or other copyright holders. Terms and conditions for use of this material are defined in the End User Agreement.

\section{www.reading.ac.uk/centaur}

\section{CentAUR}

Central Archive at the University of Reading 
Reading's research outputs online 
Dodd, H.F., Hudson, J.L., Williams, T., Morris, T., Lazarus, R.S., Byrow, Y. (2014). Anxiety and Attentional Bias in Preschool-Aged Children: An Eyetracking Study. Journal of Abnormal Child Psychology. Advance online publication. doi: 10.1007/s10802-014-9962-x

This is the author's version of the manuscript. The final, published version is available online at: http://link.springer.com/article/10.1007/s10802-014-9962-x

Corresponding author: Helen F Dodd: h.f.dodd@reading.ac.uk 


\begin{abstract}
Extensive research has examined attentional bias for threat in anxious adults and school-aged children but it is unclear when this anxiety-related bias is first established. This study uses eyetracking technology to assess attentional bias in a sample of 83 children aged 3 or 4 years. Of these, 37 (19 female) met criteria for an anxiety disorder and 46 (30 female) did not. Gaze was recorded during a free-viewing task with angry-neutral face pairs presented for $1250 \mathrm{~ms}$. There was no indication of between-group differences in threat bias, with both anxious and non-anxious groups showing vigilance for angry faces as well as longer dwell times to angry over neutral faces. Importantly, however, the anxious participants spent significantly less time looking at the faces overall, when compared to the non-anxious group. The results suggest that both anxious and non-anxious preschool-aged children preferentially attend to threat but that anxious children may be more avoidant of faces than non-anxious children.
\end{abstract}

Keywords: Attentional bias; Faces; Anxiety; Avoidance; Vigilance; Child 
Anxiety disorders are one of the most common mental health problems affecting children (Costello, Mustillo, Erkanli, Keeler, \& Angold, 2003). Increasingly, it is recognised that even preschool-aged children experience clinically significant anxiety (Egger \& Angold, 2006). Although some progress has been made in adapting Cognitive Behavioural Therapy for young children with anxiety disorders (Hirshfeld-Becker et al., 2010), relatively little is understood about the cognitive aspects of anxiety in these children.

Cognitive models of anxiety propose that attentional biases to threat-related stimuli play a role in the aetiology and maintenance of disorder (e.g., Mathews \& MacLeod, 1994). The nature of anxiety-related biases in attention has been debated extensively, with two hypotheses dominating: the vigilance-avoidance hypothesis and the attention maintenance hypothesis (Weierich, Treat, \& Hollingworth, 2008). The vigilance-avoidance hypothesis posits that anxious individuals initially orient more rapidly to threat-relevant stimuli and that this is followed by attentional avoidance of threat (Mogg, Bradley, Miles, \& Dixon, 2004). Alternatively, the maintenance hypothesis posits that anxiety is characterised by maintained attention to threat. Specifically, it is proposed that anxious individuals may have particular difficulty disengaging attention from threatening stimuli (Fox, Russo, Bowles, \& Dutton, 2001). Weierich et al. (2008) highlight that these hypotheses are not necessarily mutually exclusive; vigilance could be followed by an initial difficulty disengaging attention and then overt attentional avoidance.

One of the most frequently used behavioural tasks for examining attentional bias in anxiety is the dot-probe task (MacLeod, Mathews, \& Tata, 1986). In this task, participants are asked to respond to a target that follows emotional stimuli and reaction time is recorded. Reaction time to target is used to determine the location of participants' attention at the point of target onset. As such, the dot-probe task provides a snapshot of spatial attention at the point when the target is presented. Stimulus display times vary across studies but $500 \mathrm{~ms}$ is 
most frequently used as a proxy for vigilance and 1250ms has been used to examine maintained attention over a longer period (Koster, Verschuere, Crombez, \& Van Damme, 2005). Although there are limitations to this type of behavioural measure of attentional bias, there is evidence from this task and others, including the stroop task, that adults with elevated anxiety exhibit an attentional bias for threat (Bar-Haim, Lamy, Pergamin, BakermansKranenburg, \& van Ijzendoorn, 2007).

Anxiety-related attentional biases have also been examined in children but findings have been less consistent, with some studies finding a bias towards threat (Taghavi, Dalgleish, Moradi, Neshat-Doost, \& Yule, 2003) and others finding avoidance of threat (Stirling, Eley, \& Clark, 2006), or disorder specific patterns of vigilance and avoidance (Waters, Bradley, \& Mogg, 2013). In a recent review Shechner et al. (2012) highlighted that two studies have examined anxiety-linked attentional orienting in children younger than 5 years (Perez-Edgar et al., 2010; Perez-Edgar et al., 2011), and only one of these has examined attention to threat stimuli specifically (Perez-Edgar et al., 2011). Both of these studies focused on the temperament Behavioural Inhibition (BI), which is characterised by avoidance of novelty and a tendency to withdraw from unfamiliar people and situations (Garcia-Coll, Kagan, \& Reznick, 1984). BI is linked to anxiety disorders later in childhood (Hudson \& Dodd, 2012). Using a novelty-orienting task (Perez-Edgar et al., 2010) and the dot-probe task (Perez-Edgar et al., 2011) respectively, neither study found evidence for a cross-sectional association between BI and attentional orienting but both found that attentional orienting moderated the association between BI and anxiety later in life. Shechner and colleagues (2012) conclude that these studies provide initial evidence that the relation between anxiety and attentional orienting may not be present before children reach school age and that the anxiety-attention association may vary across development. This conclusion is somewhat supported by recent research that found an association between BI and 
attentional bias for angry faces in slightly older children using a visual search task (aged 4-7 years; LoBue \& Pérez-Edgar, 2014).

One of the reasons for the lack of attentional bias research with young children is that the tasks used to measure bias are limited. Recent findings indicate that reaction time measures of attentional bias, including the dot-probe and visual search task are not reliable in children aged 9 years (Brown et al., 2014). Given that children younger than this will have slower motor functioning, it seems unlikely that reaction time tasks can sensitively measure attentional bias in young children (Garner, 2010). In addition, these behavioural tasks can provide only limited insight into the specific components of attention that underpin bias.

In response to some of the limitations with reaction-time based measures of bias, research has begun to use eyetracking to assess attentional bias in adults and older children. The most common paradigm used is a free viewing task, in which participants are shown stimuli that vary in valence and gaze is recorded to capture natural viewing behaviour. This paradigm can be used to examine vigilance for threat and maintenance of attention to threat, with maintenance sometimes divided into initial maintenance and maintained attention to threat (see Armstrong \& Olatunji, 2012). A recent meta-analysis of studies using eyetracking to assess anxiety-linked attentional biases concluded that anxious adults exhibit vigilance for threat but no bias in initial maintenance of attention to threat (Armstrong \& Olatunji, 2012). There were not enough studies to examine maintenance of attention to threat or to report findings for child anxiety specifically.

To our knowledge, only four studies have used eyetracking to examine attentional bias in anxious children (Gamble \& Rapee, 2009; In-Albon, Kossowsky, \& Schneider, 2010; Seefeldt, Kramer, Tuschen-Caffier, \& Heinrichs, 2014; Shechner et al., 2013). All of these studies used a free-viewing paradigm, recording gaze whilst pairs of emotional stimuli were presented. The findings are somewhat inconsistent across studies but in general there is only 
modest support for an anxiety-related vigilance bias in anxious children; In-Albon et al. (2010) and Gamble and Rapee (2009) both found no vigilance bias in child participants, Seefeldt et al. (2014) found a vigilance bias in socially anxious children only when state anxiety was elevated via an experimental manipulation, and Shechner et al. (2013) found a vigilance bias but their sample included participants aged up to 17 years.

Similarly, to date, there is little indication of a bias in maintained attention in child anxiety, with three of the four studies conducted with children failing to find differences between clinically anxious children and controls on maintained attention to emotional faces (Gamble and Rapee, 2009; Seefeldt et al., 2014; Shechner et al.. 2013). Importantly, there is some indication in two studies that both anxious and non-anxious children may preferentially attend to threatening stimuli (Gamble and Rapee, 2009; Seefeldt et al., 2014). None of the studies using eyetracking with children examined initial maintenance of attention.

The aim of the present research is to use a novel child-friendly eyetracking task to assess the relation between anxiety and threat-related attentional bias in preschool-aged children. This is the first study to assess threat-related attentional bias and anxiety disorders specifically in children younger than 5 years. As in previous child eyetracking research, vigilance and maintained attention are examined. In addition, to be consistent with adult research using free-viewing paradigms, initial maintenance of attention is also examined. The following hypotheses are evaluated: 1) anxious children will be vigilant for angry faces over neutral faces; 2) this vigilance bias will differ between groups, with anxious children exhibiting greater vigilance than non-anxious children; 3) anxious children will exhibit prolonged initial maintenance of attention to threat, as compared to neutral stimuli; 4) this bias in initial maintenance will differ between groups, with anxious children exhibiting a greater bias for angry faces than non-anxious participants; 5) anxious participants will attend to angry faces for longer than neutral faces across the entire trial; 6) this bias in maintained 
attention to threat will differ between groups such that anxious participants exhibit a greater bias for angry faces than non-anxious participants.

\section{Method}

\section{Participants}

Data are reported for 83 children aged 3 and 4 years, 37 who met criteria for an anxiety disorder (Anx) and 46 who did not (NAnx). Age, sex and diagnostic information, are shown in Table 1. Participants were recruited as part of a randomised control trial (RCT) of an intervention for BI children. Recruitment was via two advertisements in a free magazine for parents; the first asked for shy children and the second asked for confident children. The advert for shy children also stated "Some families will also receive a program of five onehour sessions that focus on using the parent-child relationship to help shy children become more confident". Parents who responded completed the approach scale of the Short Temperament Scale for Children (STSC; Sanson, Smart, Prior, Oberklaid, \& Pedlow, 1994) over the phone. This contains seven items assessing the tendency to approach versus withdraw from novel situations and people. Only participants who scored more than $1 S D$ above or below the normative mean were invited to take part. These participants were considered BI and BUI respectively. On the basis of the Anxiety Disorders Interview Schedule, described below, 34 of the BI children met criteria for an anxiety disorder (from a total of $44 \mathrm{BI}$ children) and three BUI children met criteria for an anxiety disorder (from a total of 39 BUI children). These 37 children were included in the Anx group and all remaining children were included in the NAnx group.

An additional 95 participants consented to participate but did not complete the eyetracking task for the following reasons (approximate percentage of participants shown in brackets): time constraints (36\%); eyetracker not available (30\%); technical difficulties with the eyetracking equipment unrelated to this study (10\%); difficulty with obtaining good 
calibration (20\%); child did not assent to study procedures (4\%). There were no significant differences between participants who completed the eyetracking task and those who did not on age, sex, ethnicity, family income, maternal education level, BI group or anxiety group ( $p$ $>$.16). For those who completed the eyetracking task, the majority $(66 \%)$ of participants identified as Oceanic, with $11 \%$ Asian and $21 \%$ European. The majority of mothers had a degree (75\%), 94\% came from two-parent families and average income was high (> AU\$80,000 per annum). There were no significant differences between anxiety groups on these variables $(p>.1)$.

\section{Measures and apparatus}

Anxiety Disorders Interview Schedule - Parent version. Mothers were interviewed using the ADIS-P (Silverman \& Albano, 1996). Previous research has shown that the ADIS-P can be used to reliably diagnose anxiety disorders in preschool-aged children (Rapee, Kennedy, Ingram, Edwards, \& Sweeney, 2005). Diagnoses and Clinical Severity Ratings (CSRs) on a scale of zero to eight were assigned by psychology graduate students given comprehensive training and supervision ${ }^{1}$. The ADIS interview was conducted, using the skip rules and additional supplements, as described in the manual. As only parents were interviewed, the information given by parents, including interference ratings, informed diagnoses based on the criteria set out by the DSM-IV (American Psychiatric Association, 1994) and CSR ratings. Diagnoses were only considered 'clinical' if the CSR was four or greater (causing clinically significant interference). To give an indication of the types of anxiety seen in the sample, a breakdown of diagnoses is shown in Table 1 . There is some question about the differentiation of specific types of anxiety disorder at this young age, thus participants were grouped according to whether they met criteria for any anxiety disorder. To check the reliability of this group assignment, 40 interviews were selected at random and second-coded by an independent interviewer from video; this interviewer also received the 
training described. Reliability for the presence of a clinical anxiety disorder was excellent $($ Kappa $=.95)$.

\section{Eyetracking Apparatus}

Monocular eye-movements were recorded from the right eye using an Eyelink1000 (SR Research Ltd., Mississauga, Ontario, Canada) in remote mode. In this mode, eyemovements are recorded with an average accuracy of $0.5^{\circ}$ and at a sampling rate of $500 \mathrm{~Hz}$. The task was presented on a DELL 19" LCD monitor with pixel resolution 1024x768.

\section{Stimuli}

Sixteen images were selected from the Radboud Faces Database (Langner et al., 2010) an angry and neutral expression for each of eight child actors (four female). Child actors were used in light of evidence that adult faces are particularly salient to children, regardless of emotional expression (Benoit, McNally, Rapee, Gamble, \& Wiseman, 2007) and recent recommendations that attentional bias research with children should use child face stimuli (Shechner et al., 2013). Grey-scale images were edited to show only facial features, measured 200x280 pixels and were presented such that the centre of each image was 200 pixels to the left/right of the centre of the screen. The centre points of the two images were separated by a visual angle of $\approx 11^{\circ}$.

The eyetracking task was programmed using SR Research ExperimentBuilder and comprised three blocks of 16 trials. Each trial began with a fixation screen displaying a cartoon hippo measuring 53x59 pixels centrally (approx. $1.5^{\circ} \times 1.7^{\circ}$ ). Once participants had fixated on the hippo for 150ms the experimental screen was displayed. Each experimental screen included two images depicting the same actor displaying an angry and a neutral face. A pair of images for each actor was presented two times per block, with image position counterbalanced. Trials were randomised within blocks. The fixation hippo was visible throughout the trial. Each experimental screen was displayed for $1250 \mathrm{~ms}$, to keep the task 
short for participants whilst being consistent with previous research on attentional bias in anxiety (Koster et al., 2005). A blank white screen followed each trial for 1.5s.

\section{Procedure}

This research was approved by the University ethics committee and has therefore been performed in accordance with the ethical standards laid down in the 1964 Declaration of Helsinki and its later amendments. Parents provided informed consent for their child to participate and children assented to the study procedures. This research was conducted as part of baseline assessments for the RCT. Families attended a two-hour session during which observations of children's behaviour and parenting took place as well as the tasks reported here.

For the eyetracking task, participants were told that they were going to play a game on a computer that would track where they looked. They were shown a large image of the hippo and told that 'Mr Hippo' would be playing hide and seek so sometimes he would be on the screen and sometimes he wouldn't be. Participants were told that there would also be some faces on the screen, but their job was just to look at Mr Hippo when he was on the screen. Participants sat on a chair $60 \mathrm{~cm}$ away from the display screen and the target sticker required for remote tracking on the Eyelink1000 was placed on their forehead. Five point calibration and validation was used and the experiment began only when average error across the five points was less than $1^{\circ}$, with a maximum error of $1.5^{\circ}$ (defined as good calibration by the eyelink1000 software). Gaze contingency was used such that each trial only began when a fixation of at least 150ms was detected on the hippo. If a fixation was not detected, calibration and validation procedures were repeated and the gaze contingent fixation hippo was displayed again. This procedure ensured that good calibration was maintained throughout the experiment and that missing data was minimised. The experimenter sat with the child throughout the task. At the end of each block participants took a break. After each 
break, calibration and validation procedures, described above, were repeated. Whilst children completed the eyetracking task, their mother completed the ADIS-P interview with a second experimenter.

\section{Data preparation and analysis plan}

Eyetracking data were extracted using SR Research Dataviewer. For the experimental trial period, three interest areas (IAs) were created around the two face images and the fixation hippo, and fixations $<100 \mathrm{~ms}$ were removed. A number of initial checks were conducted to ensure the integrity of the eytracking data. First, the number of trials with fixations recorded was examined. This ranged from 46-48 trials, with a mean of 47.72 trials (99.4\% of trials), showing that the eyetracker recorded gaze consistently across trials. In addition, average dwell time recorded per trial was calculated. This indicated that fixations were recorded for the majority of trial time (on average $1131 \mathrm{~ms}, 91 \%$ of trial time); As a final check, given the task, one would expect total dwell time to the central fixation hippo, which measured approximately $1.5^{\circ} \times 1.7^{\circ}$, to be a significant proportion of total dwell time. Examination of dwell time confirmed this; fixations in this interest area accounted for $49 \%$ of total dwell time. The groups were closely matched on number of trials with gaze recorded, overall dwell time recorded, and dwell time to central fixation (see Table 2 for descriptive statistics), indicating that the quality of the eyetracking data was consistent across groups. Furthermore, both groups fixated on at least one face on a majority of trials. However, Anx participants fixated on a face on slightly less trials $(M=34.92, S D=9.70)$ than the NAnx participants $(\mathrm{M}=38.48, \mathrm{SD}=7.20)$, this difference approached significance, $t(81)=1.92, p$ $=.059, d=0.42$.

For the main analyses comparing angry and neutral faces, three variables were extracted based on those used in previous research (Armstrong \& Olatunji, 2012; Shechner et al., 2013). 
1. Initial vigilance: As a measure of vigilance, the proportion of trials in which the angry face was fixated upon before neutral face was calculated by dividing the number of trials in which the first face fixated upon was an angry face by the number of trials in which at least one face was fixated upon.

2. Initial maintenance: As a measure of initial maintenance of attention to emotional faces, the average length of the first fixation to an angry face was calculated by dividing the total dwell time of first fixations to an angry face by the number of trials where the first fixation was to an angry face. The equivalent was then calculated for neutral faces.

3. Maintained attention: To further measure maintained attention, average dwell time to angry and neutral faces was calculated by summing total dwell time to each image type across the experiment and dividing by the total number of trials (48).

\section{Results}

Means and standard deviations for all eyetracking variables are shown in Table 2.

\section{Initial vigilance: Probability of first fixation to angry}

Two one-sample t-tests were conducted to examine whether the probability of first fixation to angry differed from chance for the two groups separately. This analysis was significant for both the Anx group, $\mathrm{t}(36)=4.09, p<.001$, and NAnx group, $t(45)=5.76, p<$ .001. For both groups, the mean scores indicate that participants were more likely to fixate on the angry face before the neutral face. A between-groups t-tests showed that there was no significant between-group difference in probability of first fixation to angry, $t(81)=.66, p=$ $.51, d=0.15$.

\section{Initial maintenance: Length of first fixation}

The length of the first fixation to a face was examined using a mixed ANOVA with emotion (angry, neutral) and group (Anx, NAnx). There was no significant effect of emotion, $F(1,81)=0.73, p=.40, \eta_{\mathrm{p}}^{2}=.009$, and no significant interaction, $F(1,81)=.04, \mathrm{p}=.84, \eta_{\mathrm{p}}{ }^{2}$ 
$=.001$, but the effect of group was significant, $F(1,81)=6.19, p=.015, \eta_{\mathrm{p}}{ }^{2}=.07$, with Anx participants having shorter first fixations to faces than NAnx participants.

\section{Maintained attention: Dwell time}

To examine maintained attention, a two-way mixed ANOVA with group (Anx, NAnx) and emotion (angry, neutral) was conducted. There was a significant main effect of group, $F(1,81)=9.01, p=.004, \eta_{\mathrm{p}}{ }^{2}=.1$, and emotion $F(1,81)=83.15, p<.001, \eta_{\mathrm{p}}{ }^{2}=.51$, but no group $\mathrm{x}$ emotion interaction, $F(1,81)=1.29, p=.26, \eta_{\mathrm{p}}{ }^{2}=.02$. This indicates that both groups fixated for longer on the angry faces than the neutral faces and that the Anx group spent less time fixated on the faces than the NAnx group, but this was not moderated by the emotion displayed. Note that an identical pattern of results is seen when the length of the first fixation to a face is subtracted from overall dwell time to faces, indicating that this result is not driven simply by the first fixation data.

To examine where participants were looking when they weren't looking at the faces, two additional t-tests were conducted. The groups did not differ significantly on average dwell time to the central fixation, $t(81)=1.55, p=.12, d=0.34$, but the Anx group fixated on blank areas of the screen for significantly longer than the NAnx group, $t(81)=2.26, p=.027$, $d=0.50$. This between group difference can be seen in Figure 1, which shows average dwell time to faces, the fixation hippo and to blank areas of the screen by group. ${ }^{2}$

\section{Comparison with dot-probe results: $500 \mathrm{~ms}$ point}

To facilitate comparison with previous dot-probe research, the participants' fixations at 500ms after stimulus onset were examined. On average, participants were fixated on one of the faces at the $500 \mathrm{~ms}$ point on 23 of 48 trials (49.5\%). Figure 2 shows the number of trials participants in the Anx and NAnx groups were fixated on the angry and neutral image at the 500ms point. A two-way ANOVA with group (Anx, NAnx) and emotion (angry, neutral) was conducted. There was a significant main effect of group, $F(1,81)=5.89, p=.017, \eta_{\mathrm{p}}{ }^{2}=.07$, 
and emotion $F(1,81)=33.19, p<.001, \eta_{\mathrm{p}}{ }^{2}=.29$, but no group $\mathrm{x}$ emotion interaction, $F(1$, $81)=.32, p=.57, \eta_{\mathrm{p}}{ }^{2}<.01$. This suggests that both groups were more likely to be fixated on the angry than neutral face but that the anxious participants were less likely to be fixated on either face.

\section{Discussion}

The aim of this research was to examine the association between threat-related attentional biases and anxiety in preschool-aged children. The results provided only limited support for the hypotheses. As hypothesised, the anxious participants were vigilant for angry faces, relative to neutral faces, and spent longer fixated on angry than neutral faces across the full trial period. Unexpectedly however, comparable gaze patterns were found for the nonanxious children and there were no between-group differences in vigilance for angry faces. Two further hypotheses regarding initial maintenance were also not supported; there was no evidence that anxious children initially maintained attention on angry faces for longer than neutral faces or in comparison to non-anxious children. This later finding is consistent with adult eyetracking research, which has also failed to find an anxiety-related bias in initial maintenance of attention (Armstrong \& Olatunji, 2012). It seems plausible that free-viewing tasks are not ideal for detecting problems with the disengagement of attention and a more sophisticated eyetracking paradigm that requires participants to rapidly disengage and shift their attention may be required.

Although limited research has examined anxiety-related attentional bias to threat in very young children, the one previous study that focused specifically on preschool-aged children (Perez-Edgar et al., 2011) failed to find an association between attention to threat and BI, which is linked to anxiety risk (e.g., Chronis-Tuscano et al., 2009; Hudson \& Dodd, 2012). On the basis of these findings, Shechner et al. (2012) suggest that there may be agespecificity in the anxiety-attention association. Similarly, Field and Lester (2010) review the 
evidence of attentional to threat in children, taking a developmental perspective, and conclude that preferential allocation of attention to threat appears to be normative in early childhood and that anxiety-related differences emerge with development. The comparable attention to threat found in our anxious and non-anxious groups is in keeping with this hypothesis. Furthermore, two previous eyetracking studies with anxious children have also found comparable attention to threat stimuli in anxious and non-anxious groups; Seefeldt et al., (2014) reported vigilance for angry over neutral faces in both anxious and non-anxious children aged 8-12 years and Gamble and Rapee (2009) found that both anxious and nonanxious children attended to angry faces for longer than neutral faces within the first 2 seconds of stimulus presentation.

If development does affect the anxiety-attention association and an anxiety-linked bias emerges with age, this may explain some of the inconsistency in previous attentional bias research with children; studies often include a broad age-range of children and the relative balance of older and younger children could affect the conclusions of the research. Significantly more research is required before any conclusions can be drawn about the development of anxiety-linked attentional bias but research that focuses on narrow age ranges will be valuable. Furthermore, longitudinal work examining how the association between preferential attention to threat and anxiety changes across development and which developmental factors in particular (e.g., inhibition; Kindt, Bierman, \& Brosschot, 1997), might moderate this relation will be of interest.

It is of course important to consider that between-group differences in attentional bias may be found using a different paradigm. For example, there is some indication that anxietyrelated biases are clearest when threat stimuli are less overtly threatening or when schematic images are used rather than photographs of faces (Koster, Crombez, Verschuere, Van Damme, \& Wiersema, 2006; Ohman, Lundqvist, \& Esteves, 2001; Watts \& Weems, 2006). 
Thus, it is possible that an anxiety-linked attentional bias would be found in young children if less provoking stimuli were used. Furthermore, the lack of between group differences in attention to threat as measured by eyetracking does not preclude the possibility of betweengroup differences that could be detected at the neural level. Electroencephalography (EEG) and/or functional magnetic resonance imaging (fMRI) may be able to detect subtle attentionrelated differences between anxious and non-anxious groups that cannot be captured by gaze alone. Furthermore these methods may provide additional insights into the neural processes underpinning attentional bias. For example, MacNamara and Hajcak (2010) measured ERPs as well as performance accuracy whilst anxious adults completed an image-matching task, which involved the presentation of aversive and neutral images presented in attended and unattended locations. The ERP and accuracy data provided complementary insights into distinct aspects of attentional bias. Similarly, youth with post traumatic stress symptoms have greater activation than controls in several brain regions implicated in attention to emotional images, including the medial prefrontal cortex, the amygdala/hippocampus, insula and ventrolateral prefrontal cortex and less activation than controls in the dorsolateral prefrontal cortex (Garrett et al., 2012). These anxiety-related differences would not necessarily be detected by eyetracking or a behavioural reaction time task. There are, therefore, numerous ways the present research could be extended to further explore the attention-anxiety relation in young children.

\section{Avoidance of faces in anxious participants}

Importantly, an unexpected but clear group difference in attention to faces in general was found; anxious participants fixated on a face on slightly less trials, spent significantly less time looking at the faces and had significantly shorter first fixations to the faces, irrespective of emotion. This suggests that anxious participants were avoidant of face stimuli, relative to non-anxious participants, at all stages of attentional processing. This finding must 
be interpreted with caution as it was unexpected, but one explanation is that the anxious participants interpreted the neutral face as threatening. The cognitive-motivational account states that anxiety affects the reactivity of a 'valence evaluation system' such that mild or ambiguous threat cues are more readily appraised as threatening, and that the output of this system then affects the allocation of attention (Mogg \& Bradley, 1998). Following this, the anxious participants may have appraised the neutral faces as threatening and subsequently avoided both 'threatening' faces more than non-anxious participants.

Whilst this avoidance of face stimuli initially seems inconsistent with previous research, it is possible that avoidance of faces was present but missed in some previous studies. For example, in eyetracking research where a bias score is calculated by comparing attention to two stimulus categories, absolute differences in dwell time are masked (e.g. Gamble \& Rapee, 2009; Seefeldt et al., 2014). Similarly, in the dot-probe task, relative attention to threat-related stimuli compared to non-threat stimuli is calculated and avoidance of both would be missed. The present results seem reasonably compatible with Huijding, Mayer, Koster and Muris (2011) who found that participants with spider fear made more nontarget fixations than participants without spider fear when the target was a spider, suggesting avoidance. Similarly, LoBue and Perez-Edgar (2014) reported that shy participants were slower than non-shy participants to locate angry faces in a visual search task. It is plausible that this could have been caused by subtle avoidance of the face stimuli by shy participants.

There are a number of points that must be taken into account when considering this between-group difference in attention to faces. First, most of the anxious participants were also behaviourally inhibited, which is characterised by avoidance of novelty. Therefore this avoidance of faces may be specific to this subgroup of anxious children. It was not possible to tease apart the effects of BI and anxiety in the present study as only three BUI participants met criteria for an anxiety disorder. However, it is important to consider that a substantial 
proportion of anxious preschool children are also BI, so the present sample is not unrepresentative of the population of anxious preschoolers. In addition, understanding cognition in this group of children is important because we know from previous research that these children are particularly at risk for ongoing problems with anxiety (Hudson \& Dodd, 2012; Perez-Edgar et al., 2011), particularly social anxiety (e.g., Chronis-Tuscano et al., 2009).

A related consideration is that a substantial proportion of the anxious group (59\%) met criteria for Social Anxiety Disorder so it is possible that avoidance of faces is a feature of early onset Social Anxiety Disorder rather than anxiety disorders more generally. The present study was not designed to address the diagnostic specificity of attentional bias; given that the sample were BI, there is an over-representation of social anxiety, and there is some question about the stability and specificity of individual anxiety diagnoses in preschool-aged children. However, preliminary analyses ${ }^{2}$ suggest it is unlikely the results were specific to participants with Social Anxiety Disorder. This question could be considered in further research conducted with a clinical sample of children with a range of anxiety disorders. A final point is that participants were told their task was to look at the fixation hippo; it is therefore possible that less looking time at the faces reflects superior task performance or more compliance by the anxious participants. In this case, the task may indirectly be measuring attentional control (Eysenck \& Derakshan, 2011). A significant group difference was, however, found on dwell time to blank areas of the screen rather than dwell time to fixation hippo. Although this indicates that an attentional control explanation is unlikely, it would be useful to replicate this experiment using a modified version of the task in which the hippo leaves the screen when the faces are presented.

\section{Strengths and limitations}


This research provides an important first step towards understanding allocation of attention in young children with anxiety disorders. The study has a number of strengths including the detailed clinical assessment of anxiety and the relatively large sample size as well as the novel, developmentally appropriate eyetracking task. The paradigm was kept intentionally simple to ensure it was feasible for young children to complete. As such, the stimulus presentation times are relatively short in comparison to other eyetracking studies and it remains possible that other between group differences may emerge over longer presentation times. In addition, to minimise the number of trials, only angry-neutral face pairs were presented. This limits the conclusions that can be drawn from the present results. The preferential allocation of attention toward angry faces found for the sample as a whole could be driven by the threatening nature of angry faces, the emotional nature of angry faces or avoidance of ambiguity, as present in neutral faces. An adapted version of this task with longer stimulus presentation times and positive as well as negative emotions is currently being developed to explore these possibilities but we remain sensitive to the demands that can reasonably be placed on a young child.

To further minimise task burden, a free-viewing task was used rather than combining eyetracking with a dot-probe paradigm. However, there was a clear gaze preference for angry over neutral faces at the $500 \mathrm{~ms}$ point in both groups of participants, suggesting that a dotprobe version of this task would also have indicated a significant attentional preference towards angry faces for both groups. Finally, we removed fixations with a duration shorter than $100 \mathrm{~ms}$ to be consistent with previous eyetracking research in clinical psychology (e.g., Gamble \& Rapee, 2009) and eyetracking research with preschool aged children (Nuske, Vivant, \& Dissanayake, 2014). However, it is not clear how much information children of this age can extract in $100 \mathrm{~ms}$; a shorter or longer fixation duration cut-off may be more appropriate for this age-group. There is some evidence from reading research that preschool 
children have a smaller perceptual span and longer fixation times than adults (Rayner, 1986) but it isn't clear how this applies to the present task. Basic cognitive developmental research that provides clear guidance for eyetracking research with children would be most beneficial.

\section{Clinical implications}

With regards the clinical significance of the between-group differences found, the effect sizes suggest a medium effect, with group accounting for between $7 \%$ and $10 \%$ of the variance in gaze behaviour. It is feasible that this relatively subtle between-group difference could have clinical significance for the development of anxiety over time if it is: a) present

early in a child's life; b) stable over time; and c) representative of their attentional orienting across multiple real-life contexts. The present findings provide preliminary support for the first of these statements but statements $b$ and $c$ represent unanswered questions for further work in this field.

It is also of relevance, whilst considering the clinical implications of the findings, to discuss Attentional Bias Modification (ABM). The aim of ABM research is to examine whether attentional biases can be changed and whether change in bias could lead to improvements in anxiety symptoms or emotional reactivity to stress. To date, ABM research has yielded some promising results, but findings overall have been mixed. Whilst we are not yet in a position to consider using ABM with young children, research that establishes the nature of anxiety-related biases in young children, such as the present research, is important for guiding future decisions about whether it may be appropriate to attempt to change biases in this population. 


\section{Acknowledgements}

This research was conducted as part of an Australian Research Council funded project (DP0878609 \& DP0342793) held by Professor Hudson. Thank you to the interns and students who helped with stimuli, data collection and coding for this project, including Laurie Monier, Anni Kuusik, and Irma Knuistingh. Thanks also to Emily Connaughton and Eugene McSorely for their help with the eye-tracking data, Suzannah Ravenscroft for her comments on an earlier draft and to the families who participated.

\section{Conflict of Interest}

The authors have no conflicts of interest to declare. 


\section{References}

American Psychiatric Association. (1994). Diagnostic and statistical manual of mental disorders (4th ed.). Washington DC: American Psychiatric Association.

Armstrong, T., \& Olatunji, B. O. (2012). Eye tracking of attention in the affective disorders: a meta-analytic review and synthesis. Clinical psychology review, 32, 704-723. doi: 10.1016/j.cpr.2012.09.004

Bar-Haim, Y., Lamy, D., Pergamin, L., Bakermans-Kranenburg, M .J., \& van Ijzendoorn, M. H. (2007). Threat-related attentional bias in anxious and nonanxious individuals: A meta-analytic study. Psychological bulletin, 133, 1-24. doi: http://dx.doi.org/10.1037/0033-2909.133.1.1

Beard, C., Sawyer, A. T., \& Hofmann, S. G. (2012). Efficacy of Attention Bias Modification Using Threat and Appetitive Stimuli: A Meta-Analytic Review. Behavior therapy, 43, 724-740. doi: http://dx.doi.org/10.1016/j.beth.2012.01.002

Benoit, K. E., McNally, R. J., Rapee, R.M., Gamble, A. L., \& Wiseman, A. L. (2007). Processing of Emotional Faces in Children and Adolescents With Anxiety Disorders. Behaviour change, 24, 183-194. doi: doi:10.1375/bech.24.4.183

Brown, H. M., Eley, T. C., Broeren, S., MacLeod, C., Rinck, M., Hadwin, J. A., \& Lester, K. J. (2014). Psychometric properties of reaction time based experimental paradigms measuring anxiety-related information-processing biases in children. Journal of anxiety disorders, 28, 97-107. doi: http://dx.doi.org/10.1016/j.janxdis.2013.11.004

Chronis-Tuscano, A., Degnan, K. A., Pine, D. S., Perez-Edgar, K., Henderson, H. A., Diaz, Y., .. . Fox, N. A. (2009). Stable Early Maternal Report of Behavioral Inhibition Predicts Lifetime Social Anxiety Disorder in Adolescence. Journal of the american academy of child and adolescent psychiatry, 48, 928-935. doi:

10.1097/CHI.0b013e3181ae09df 
Costello, E., Mustillo, S., Erkanli, A., Keeler, G., \& Angold, A. (2003). Prevalence and Development of Psychiatric Disorders in Childhood and Adolescence. Archives of general psychiatry, 60, 837-844. doi: 10.1001/archpsyc.60.8.837

Egger, H. L., \& Angold, A. (2006). Common emotional and behavioral disorders in preschool children: presentation, nosology, and epidemiology. Journal of child psychology and psychiatry, 47, 313-337.

Eysenck, M. W., \& Derakshan, N. (2011). New perspectives in attentional control theory. Personality and individual differences, 50, 955-960. doi: http://dx.doi.org/10.1016/j.paid.2010.08.019

Field, A. P., \& Lester, K. J. (2010). Is there room for 'development' in developmental models of information processing biases to threat in children and adolescents? Clinical child and family psychology review, 13, 315-332. doi: 10.1007/s10567-010-0078-8

Fox, E., Russo, R., Bowles, R., \& Dutton, K. (2001). Do threatening stimuli draw or hold visual attention in subclinical anxiety? Journal of experimental psychology: general, 130, 681-700. doi: 10.1037/0096-3445.130.4.681

Gamble, A. L., \& Rapee, R. M. (2009). The time-course of attentional bias in anxious children and adolescents. Journal of anxiety disorders, 23, 841-847. doi: http://dx.doi.org/10.1016/j.janxdis.2009.04.001

Garcia-Coll, C, Kagan, J, \& Reznick, J. (1984). Behavioral Inhibition in young children. Child development, 55, 1005-1019. doi: 10.2307/1130152

Garner, M. (2010). Assessment of attentional bias using the dot-probe task in anxious children and adolescents. In J. H. A. P. Field (Ed.), Information processing biases and anxiety: a developmental perspective (pp. 77-109). Chichester: Wiley-Blackwell. 
Garrett, A. S., Carrion, V., Kletter, H., Karchemskiy, A., Weems, C. F., \& Reiss, A. (2012). Brain activation to facial expressions in youth with PTSD symptoms. Depression and anxiety, 29, 449-459. doi: 10.1002/da.21892

Hallion, L. S., \& Ruscio, A. M. (2011). A meta-analysis of the effect of cognitive bias modification on anxiety and depression. Psychological bulletin, 137, 940-958. doi: 10.1037/a0024355

Hirshfeld-Becker, D. R., Masek, B., Henin, A., Blakely, L. R., Pollock-Wurman, R. A., McQuade, J, .. . J, Biederman. (2010). Cognitive behavioral therapy for 4- to 7-yearold children with anxiety disorders: a randomized clinical trial. Journal of consulting and clinical psychology, 78, 498-510. doi: 10.1037/a0019055

Hudson, J. L., \& Dodd, H. F. (2012). Informing Early Intervention: Preschool Predictors of Anxiety Disorders in Middle Childhood. PLoS ONE, 7, e42359. doi: 10.1371/journal.pone.0042359

Huijding, J., Mayer, B., Koster, E. H., \& Muris, P. (2011). To look or not to look: An eyemovement study of hypervigilance during change detection in high and low spider fearful students. Emotion, 11, 666-674.

In-Albon, T., Kossowsky, J., \& Schneider, S. (2010). Vigilance and Avoidance of Threat in the Eye Movements of Children with Separation Anxiety Disorder. Journal of abnormal child psychology, 38, 225-235. doi: 10.1007/s10802-009-9359-4

Kindt, M., Bierman, D., \& Brosschot, J. F. (1997). Cognitive Bias in Spider Fear and Control Children: Assessment of Emotional Interference by a Card Format and a Single-Trial Format of the Stroop Task. Journal of experimental child psychology, 66, 163-179. doi: http://dx.doi.org/10.1006/jecp.1997.2376

Koster, E. H., Crombez, G., Verschuere, B., Van Damme, S., \& Wiersema, J.R. (2006). Components of attentional bias to threat in high trait anxiety: Facilitated engagement, 
impaired disengagement, and attentional avoidance. Behaviour research and therapy, 44, 1757-1771. doi: http://dx.doi.org/10.1016/j.brat.2005.12.011

Koster, E. H., Verschuere, B., Crombez, G., \& Van Damme, S. (2005). Time-course of attention for threatening pictures in high and low trait anxiety. Behaviour research and therapy, 43, 1087-1098. doi: 10.1016/j.brat.2004.08.004

Langner, O, Dotsch, R, Bijlstra, G, Wigboldus, D.H.J, Hawk, S.T, \& van Knippenberg, A (2010). Presentation and validation of the Radboud Faces Database. Cognition and emotion, 24, 1377-1388. doi: 10.1080/02699930903485076

LoBue, V., \& Pérez-Edgar, K. (2014). Sensitivity to social and non-social threats in temperamentally shy children at-risk for anxiety. Developmental science, 17, 239247. doi: $10.1111 /$ desc. 12110

Lyneham, H. J., Abbott, M. J., \& Rapee, R. M. (2007). Interrater reliability of the Anxiety Disorders Interview Schedule for DSM-IV: child and parent version. Journal of the american academy of child and adolescent psychiatry, 46, 731-736. doi: 10.1097/chi.0b013e3180465a09

MacLeod, C., Mathews, A., \& Tata, P. (1986). Attentional bias in emotional disorders. Journal of abnormal psychology, 95, 15-20.

MacNamara, A., \& Hajcak, G. (2010). Distinct electrocortical and behavioral evidence for increased attention to threat in generalized anxiety disorder. Depression and anxiety, 27, 234-243. doi: 10.1002/da.20679

Mathews, A., \& MacLeod, C. (1994). Cognitive Approaches to Emotion and Emotional Disorders. Annual review of psychology, 45, 25-50. doi: doi:10.1146/annurev.ps.45.020194.000325 
Mogg, K, \& Bradley, B. P. (1998). A cognitive-motivational analysis of anxiety. Behaviour research and therapy, 36, 809-848. doi: http://dx.doi.org/10.1016/S0005$7967 \% 2898 \% 2900063-1$

Mogg, K, Bradley, B. P, Miles, F., \& Dixon, R. (2004). Time course of attentional bias for threat scenes: Testing the vigilance- avoidance hypothesis. Cognition and emotion, 18, 689-700. doi: 10.1080/02699930341000158

Mogoaşe, C., David, D., \& Koster, E. H. W. (2014). Clinical Efficacy of Attentional Bias Modification Procedures: An Updated Meta-Analysis. Journal of clinical psychology. Advance online publication. doi: 10.1002/jclp.22081

Nuske, H. J., Vivanti, G., \& Dissanayake, C. (2014). Reactivity to feaful expressions of familiar and unfamiliar children with autism: an eye-tracking pupillometry study. Journal of neurodevelopmental disorders, 6, 14-30. doi: 10.1186/1866-1955-6-14.

Ohman, A., Lundqvist, D., \& Esteves, F. (2001). The face in the crowd revisited: a threat advantage with schematic stimuli. Journal of personality and social psychology, 80, 381-396.

Perez-Edgar, K., McDermott, J. N., Korelitz, K., Degnan, K. A., Curby, T. W., Pine, D. S., \& Fox, N. A. (2010). Patterns of sustained attention in infancy shape the developmental trajectory of social behavior from toddlerhood through adolescence. Developmental psychology, 46, 1723-1730. doi: 10.1037/a0021064

Perez-Edgar, K., Reeb-Sutherland, B. C., McDermott, J. M., White, L. K., Henderson, H. A., Degnan, K. A., . . Fox, N. A. (2011). Attention biases to threat link behavioral inhibition to social withdrawal over time in very young children. Journal of abnormal child psychology, 39, 885-895. doi: 10.1007/s10802-011-9495-5 
Rapee, R. M., Kennedy, S., Ingram, M., Edwards, S., \& Sweeney, L.. (2005). Prevention and Early Intervention of Anxiety Disorders in Inhibited Preschool Children. Journal of consulting and clinical psychology, 73, 488-497. doi: 10.1037/0022-006X.73.3.488

Rayner, K. (1986). Eye movements and the perceptual span in beginning and skilled readers. Journal of experimental child psychology, 41, 211-236.

Sanson, A., Smart, D., Prior, M., Oberklaid, F., \& Pedlow, R. (1994). The Structure of Temperament from Age 3 to 7 Years: Age, Sex, and Sociodemographic Influences. Merrill-Palmer quarterly, 40, 233-252. doi: http://www.asu.edu/clas/ssfd/mpq/

Seefeldt, W. L., Kramer, M., Tuschen-Caffier, B., \& Heinrichs, N. (2014). Hypervigilance and avoidance in visual attention in children with social phobia. Journal of behavior therapy and experimental psychiatry, 45, 105-112. doi: 10.1016/j.jbtep.2013.09.004

Shamir-Essakow, G., Ungerer, J. A., \& Rapee, R. M. (2005). Attachment, behavioral inhibition, and anxiety in preschool children. Journal of abnormal child psychology, $33,131-143$.

Shechner, T., Britton, J. C, Pérez-Edgar, K, Bar-Haim, Y, Ernst, M, Fox, N. A, . . Pine, S. D. (2012). Attention biases, anxiety, and development: Toward or away from threats or rewards? Depression and anxiety, 29, 282-284. doi: 10.1002/da.20914

Shechner, T., Jarcho, J. M., Britton, J. C., Leibenluft, E., Pine, D. S., \& Nelson, E. E. (2013). Attention bias of anxious youth during extended exposure of emotional face pairs: an eyetracking study. Depression and anxiety, 30, 14-21. doi: 10.1002/da.21986

Silverman, W.K., \& Albano, A. M. (1996). The anxiety disorders interview schedule for children for DSM-IV: child and parent versions. San Antonia, TX: Psychological Corporation.

Stirling, L. J., Eley, T. C., \& Clark, D. M. (2006). Preliminary evidence for an association between social anxiety symptoms and avoidance of negative faces in school-age 
children. Journal of clinical child and adolescent psychology, 35, 431-439. doi: 10.1207/s15374424jccp3503_9

Taghavi, M. R., Dalgleish, T., Moradi, A. R., Neshat-Doost, H. T., \& Yule, W. (2003). Selective processing of negative emotional information in children and adolescents with Generalized Anxiety Disorder. British journal of clinical psychology, 42, 221230. doi: $10.1348 / 01446650360703348$

Waters, A. M, Bradley, B. P, \& Mogg, K. (2013). Biased attention to threat in paediatric anxiety disorders (generalized anxiety disorder, social phobia, specific phobia, separation anxiety disorder) as a function of 'distress' versus 'fear' diagnostic categorization. Psychological medicine, firstview, 1-10. doi: doi:10.1017/S0033291713000779

Watts, S. E., \& Weems, C. F. (2006). Associations Among Selective Attention, Memory Bias, Cognitive Errors and Symptoms of Anxiety in Youth. Journal of abnormal child psychology, 34, 838-849. doi: 10.1007/s10802-006-9066-3

Weierich, M. R., Treat, T. A., \& Hollingworth, A. (2008). Theories and measurement of visual attentional processing in anxiety. Cognition and emotion, 22, 985-1018. 
Table 1

Sex, age and number of participants with specific diagnoses within their diagnostic profile for anxious (Anx) and non-anxious (NAnx) groups.

$$
\text { Anx }(n=37) \quad \text { NAnx }(n=46)
$$

Demographics

Sex (percentage female)

Age (in months)

Only one anxiety diagnosis

Two or more anxiety diagnoses

Anxiety diagnoses

Social Anxiety Disorder

Separation Anxiety Disorder

Specific Phobia

Generalised Anxiety Disorder

Obsessive Compulsive Disorder

Post Traumatic Stress Disorder

Anxiety Disorder Not Otherwise Specified

Dysthymia/Major Depression

Attention Deficit Hyperactivity Disorder

\section{$51 \%$}

$65 \%$

$M=48.03$

$M=47.83$

$(S D=4.43)$

$(S D=3.64)$
17

20

22

13

7

3

$1^{\mathrm{a}}$

0

7

3

0
0

0

0

0

0

0

0

0

0

0

1

${ }^{a}$ Note, this participant had a primary diagnosis of Social Anxiety Disorder 
Table 2

Descriptive statistics for eyetracking variables split by anxiety group.

$\begin{array}{cc}\text { Anx } & \text { NAnx } \\ (n=37) & (n=46)\end{array}$

\section{Initial checks of eyetracking data}

Number of trials with data recorded

47.73 trials $\quad 47.72$ trials

$(0.56) \quad(0.58)$

Average dwell time recorded

$1141 \mathrm{~ms} \quad 1124 \mathrm{~ms}$

(40ms) $\quad(56 m s)$

Average dwell time to central fixation

$588.15 \mathrm{~ms} \quad 533.13 \mathrm{~ms}$

(151.42ms) (166.93ms)

Number of trials with fixation to a face

34.92

$38.38^{\wedge}$

(9.7)

\section{Hypothesis testing}

Proportion of trials fixated to angry first

$0.56(0.08) \quad 0.57(0.08)$

Average length of first fixation to faces overall

$356.16 \mathrm{~ms} \quad 398.65 \mathrm{~ms}^{*}$

(66ms) $\quad(75.21 \mathrm{~ms})$

- Angry faces

$361.21 \mathrm{~ms} \quad 402.79 \mathrm{~ms} *$

(61.08ms) (84.88ms)

- Neutral faces

$356.37 \mathrm{~ms} \quad 394.93 \mathrm{~ms}^{*}$

$(93.73 \mathrm{~ms}) \quad(77.52 \mathrm{~ms})$

Average dwell time to faces overall

$414.89 \mathrm{~ms} \quad 523.80 \mathrm{~ms} * *$

$(165.87 \mathrm{~ms}) \quad(162.79 \mathrm{~ms})$

- Angry faces

$233.48 \mathrm{~ms} \quad 295.31 \mathrm{~ms} * *$

(93.71ms) (91.96ms) 
- Neutral faces

$$
\begin{array}{ll}
181.89 \mathrm{~ms} & 229.01 \mathrm{~ms} * * \\
(81.57 \mathrm{~ms}) & (81.13 \mathrm{~ms})
\end{array}
$$

\section{Additional Analyses}

$\begin{array}{lcc}\text { Average dwell time to blank areas of the screen } & 120.37 \mathrm{~ms} & 83.73 \mathrm{~ms}^{*} \\ & (80.26 \mathrm{~ms}) & (67.62 \mathrm{~ms}) \\ \text { Number of trials fixated on a face at } 500 \mathrm{~ms} & 20.68 & 26.28 \\ & (11.04) & (9.98)^{*} \\ \text { - angry faces } & 11.71(5.98) & 14.80 \\ & & (6.23)^{*} \\ \text { - neutral faces } & 8.97(5.53) & 11.48 \\ & & (5.20)^{*}\end{array}$

Note. Between-group difference significant at: ${ }^{\wedge} p<.1 ;{ }^{*} p<.05 ; * * p<.01$. 


\section{Figure captions}

Fig.1 Group differences in average dwell time across trial split by area of interest (angry face, neutral face, central fixation hippo and blank areas of the screen). Error bars show 1SD.

Fig.2 Group differences in number of trials where participants were fixated on a face (angry/neutral) 500ms after stimulus onset. Error bars show 1SD. 


\section{Footnotes}

${ }^{1}$ Prior to conducting any interviews for the present research, all interviewers first watched at least two videos of ADIS interviews. For each, they were asked to assign diagnoses and CSRs. These were then discussed with a senior clinical psychologist, with reference to the diagnoses and CSRs given by the original interviewer. Once the clinical psychologist was satisfied with their performance, trainees observed two ADIS interviews in our clinic. Again they were asked to assign diagnoses and CSRs and these were discussed after the interview with the psychologist who had conducted the assessment. Once they had completed at least two of these and assigned diagnoses and CSRs that were equivalent to those made by the interviewing clinical psychologist, they were then observed conducting at least two interviews. Again, assigned diagnoses and CSRs were discussed with the psychologist who had observed the interview. The clinical psychologist who oversaw the training made a decision regarding the interviewers readiness to conduct interviews for the study based on their overall performance on these tasks. Note that this procedure is followed for training psychologists in our clinic, where excellent reliability is reported (Lyneham, Abbott, \& Rapee, 2007). Interviewers attended regular supervision sessions with a senior clinical psychologist where difficult diagnostic cases were discussed.

${ }^{2}$ Following a request from an anonymous reviewer, we examined whether the results were driven by participants with a diagnosis of social anxiety disorder. The significant betweengroup effects reported above for length of first fixation and dwell time were examined comparing three groups of participants: those with a diagnosis of social anxiety disorder anywhere in their diagnostic profile $(\mathrm{n}=22)$, clinically anxious participants without a diagnosis of social anxiety disorder $(n=15)$ and participants with no anxiety diagnosis $(n=44)$. These analyses also showed no group by emotion interactions and a significant main effect of group for both length of first fixation, $\mathrm{F}(2,80)=3.93, p=.023$, and dwell time, $\mathrm{F}(2,80)=$ 
$6.08, p=.003$. Follow-up t-tests comparing groups showed that the group with social anxiety disorder had shorter first fixations to faces and shorter dwell time to faces than the control comparison group but neither reached significance $(p>.08)$. The clinically anxious group without social anxiety disorder also had shorter first fixations to faces and shorter dwell time to faces than the control comparison group and both reached significance $(p<.005)$. The two anxious groups were not significantly different from each other on either dependent variable $(p>.09)$. 
Running head: ATTENTIONAL BIAS IN PRESCHOOLERS

Conflict of interest

The authors declare that they have no conflict of interest. 